\title{
MAGIC observations of the February 2014 flare of 1ES 1011+496 and measurement of the Extragalactic Background Light density
}

\author{
Adiv González Muñoz ${ }^{a *}$, Priyadarshini Bangale ${ }^{\dagger b}$, Abelardo Moralejo ${ }^{a}$, Daniel Mazin $^{c}$ \\ and levgen Vovk ${ }^{b}$ for the MAGIC Collaboration \\ ${ }^{a}$ IFAE, Barcelona, Spain. \\ ${ }^{b}$ MPI for Phyisics, Munich, Germany. \\ ${ }^{c}$ ICRR, U-Tokyo, Japan. \\ E-mail: adiv.gonzalez@fisica.unam.mx, priya@mpp.mpg.de, \\ moralejo@ifae.es, mazin@icrr.u-tokyo.ac.jp, Ievgen.Vovk@mpp.mpg.de
}

\begin{abstract}
1ES $1011+496$ is a blazar located at a redshift $\mathrm{z}=0.212$, revealed as a very-high-energy $\gamma$-ray emitter by MAGIC in 2007. In February 2014 the source underwent an unprecedented flaring episode. Following a flare alert issued by VERITAS, the MAGIC telescopes carried out an observation campaign for a total of 17 nights between February 6 and March 7, during which the source reached a peak flux of almost 14 times the flux measured by MAGIC at the time of discovery, before returning to its low state. Despite the large flux variations, the estimated intrinsic spectral shape was remarkably stable through the whole period. The average spectrum during the flare could be well measured up to a few $\mathrm{TeV}$, which makes it an ideal observation for probing the Extragalactic Background Light (EBL) through its effects on the $\gamma$-ray flux. We implemented a method similar to the one used recently in high- and very-high-energy $\gamma$-ray astronomy for this purpose, consisting in a likelihood maximization in which both the intrinsic spectral parameters of the source and the EBL density are free parameters. With this method we computed limits on the EBL density using as a template Domínguez et al. (2011) model with an additional scaling parameter. This measurement is among the most constraining ones obtained with $\gamma$-ray telescopes on a single source and strengthens the case for no significant contribution of unresolved sources to the EBL.
\end{abstract}

The 34th International Cosmic Ray Conference,

30 July- 6 August, 2015

The Hague, The Netherlands

* Now at IFUNAM, Mexico City, Mexico.

† Speaker. 


\section{Introduction}

1ES $1011+496\left(\right.$ RA: $10^{\mathrm{h}} 15^{\mathrm{m}} 04.1^{\mathrm{s}}$, DEC: $+49^{\circ} 26^{\mathrm{m}} 01^{\mathrm{s}}$ ) is an active galactic nucleus (AGN) classified as a high-frequency peaked BL Lac (HBL), located at redshift $=0.212$ [1]. It was discovered at VHE by the MAGIC Collaboration in 2007 following an optical high state reported by the Tuorla Blazar Monitoring Programme [1]. Two more multi-wavelength campaigns have been organized by MAGIC since then: the first one between March and May 2008 [2] and a second one divided in two periods, from 2011 March to April and from 2012 January to May [3]. In all previous observations (including the discovery) the source did not show evidence of flux variability within the observed periods and the observed spectra could be fitted with simple power-law functions.

The observation of a intermediate-redshift source like 1ES 1011+496 on a bright state provides a good opportunity to measure the impact of the Extragalactic Background Light (EBL) on the propagation of $\gamma$ rays over cosmological distances. The EBL is the diffuse radiation that comes from the contributions of all the light emitted by stars in the UV-optical and near infrared (NIR) bands. It also contains the infrared (IR) radiation emitted by dust after absorbing the starlight, plus a small contribution from AGNs [4]. VHE $\gamma$ rays from extragalactic sources interact with the EBL in the optical and NIR bands, producing electron-positron pairs, which causes an attenuation of the VHE photon flux measured at Earth [5].

Strict lower limits on the optical band of the EBL have been derived from galaxy counts [6, 7, 8]. At NIR wavelengths, one way to access the EBL is by large-scale anisotropy measurements $[9,10,11]$. Upper limits have been set $[12,13,14]$ under the assumptions, derived from $\gamma$-ray emission models, that the intrinsic VHE source spectra cannot be harder than a given limit (photon index $>1.5$ ), nor harder than in the $<100 \mathrm{GeV}$ range, as measured by e.g. Fermi-LAT $[15,16,17]$.

The Fermi collaboration actually measure the EBL density from LAT data [18]. The spectra from $150 \mathrm{BL}$ Lacs were fitted to log parabolae functions in the optically-thin regime $(\mathrm{E}<25 \mathrm{GeV})$ and extrapolated to higher energies, then the comparison with the actually observed spectra was used to determine the best-fit scaling factor for the optical depth from a given EBL model. They obtained a measurement of the UV component of the EBL of $3 \pm 1 \mathrm{nW} \mathrm{m} \mathrm{sr}^{-1}$.

The H.E.S.S. collaboration used a similar likelihood ratio test to measure the EBL from their AGN data [19]. The EBL absorption at VHE is expected to leave an imprint in the observed spectra, coming from a distinctive feature (an inflection point in the log flux - log E representation) between $\sim 100 \mathrm{GeV}$ and $\sim 5-10 \mathrm{TeV}$. The H.E.S.S collaboration modeled the intrinsic spectra of several AGNs using simple functions (up to 4 parameters), then applied the EBL absorption $\exp (-\alpha \times \tau(E, z))$, where the optical depth $\tau$ is scaled by a factor $\alpha$. A scan over $\alpha$ was performed to achieve the best fit to the observed VHE spectra. The no-EBL hypothesis, $\alpha=0$, was excluded at the $8.8 \sigma$ level, and the EBL flux density was constrained in the wavelength range between 0.30

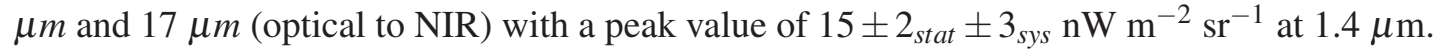

In this work we present the analysis of the extraordinary flare of 1ES 1011+496 in 2014 February-March observed by the MAGIC telescopes, and apply a technique based on [19] for measuring the EBL. 

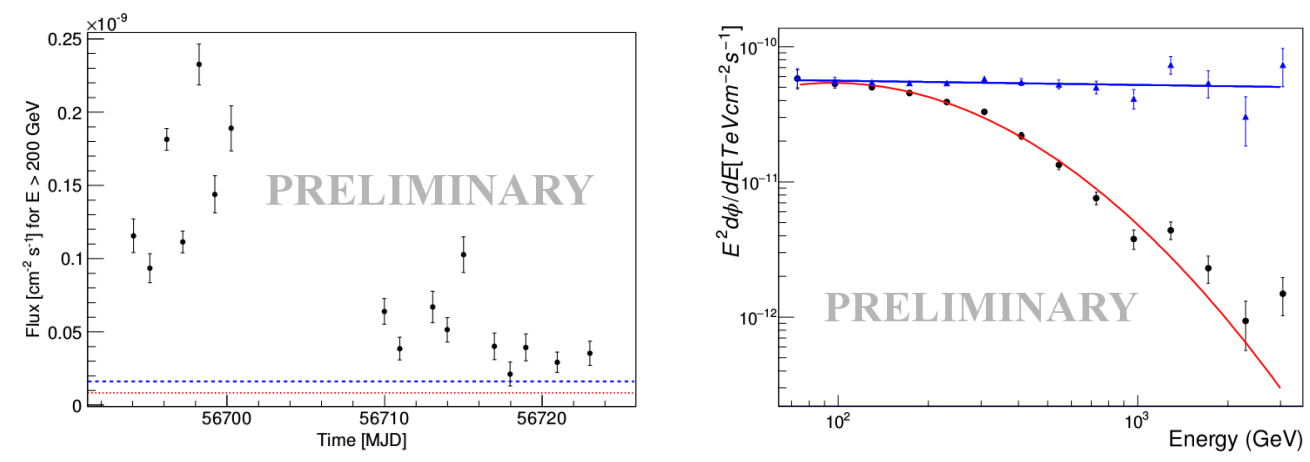

Figure 1: On the left, light curve of 1ES 1011+496 for the 17 nights between February $6^{\text {th }}$ and March $7^{\text {th }}$ 2014 above an energy threshold of $200 \mathrm{GeV}$ with a night-wise binning. The blue dashed line indicates the mean integral flux for the MAGIC observations of 2007 and the red dotted line the MWL campaign between 2011 and 2012. On the right, the average spectral energy distribution (SED) for all the observed nights. The black dots are the observed data and the blue triangles are the data after EBL de-absorption using EBL model by Domínguez et al. [23]. The red solid line indicate the fit to a log-parabola function of the observed SED whereas the blue solid line indicates the fit to a power-law function of the de-absorbed SED.

\section{Observations \& Analysis}

MAGIC is a stereoscopic system of two $17 \mathrm{~m}$ diameter IACTs situated in the Canary island of La Palma. Since the end of 2009, it has been working in stereoscopic mode with a trigger threshold of $\sim 50 \mathrm{GeV}$. During 2011 and 2012, MAGIC underwent a major upgrade [20]. With the new system the integral sensitivity achieved is of $(0.66 \pm 0.03) \%$ of the Crab nebula flux above 220 $\mathrm{GeV}$ in 50 hours at low zenith angles [21].

On February 5th 2014, VERITAS issued an alert for the flaring state of 1ES 1011+496. MAGIC performed target of opportunity (ToO) observations for 17 nights during February-March 2014 in the zenith range of $20^{\circ}-56^{\circ}$. After the quality cuts, $11.8 \mathrm{hrs}$ of good data were used for further analysis. The data were analyzed using the standard routines in the MAGIC software package for stereoscopic analysis, MARS [22].

\section{Results}

After analysis cuts, 3219 events with a expected background of 712 events was recorded around the source direction for reconstructed energies $>63 \mathrm{GeV}$.

Fig. 1 (left) shows the night by night $\gamma$-ray light curve for energies $\mathrm{E}>200 \mathrm{GeV}$ between February $6^{\text {th }}$ and March $7^{\text {th }} 2014$. The emission in this period had a high night-to-night variability, reaching a maximum of $(2.3 \pm 0.1) \times 10^{-10} \mathrm{~cm}^{-2} \mathrm{~s}^{-1}$. No significant intra-night variability was observed.

The averaged observed spectrum for the whole data could not be well fitted to a log-parabola function (see Fig. 1 right) with a probability of $0.002\left(\chi^{2}=30.7 / 12\right)$. The photon index is $\Gamma=$ $2.8 \pm 0.1$, curvature index $\beta=1.0 \pm 0.1$ and normalization factor at $E_{0}=250 \mathrm{GeV} f_{0}=(3.6 \pm$ $0.1) \times 10^{-11} \mathrm{~cm}^{-2} \mathrm{~s}^{-1} \mathrm{TeV}^{-1}$. The de-absorbed spectrum using the EBL model by [23] can be 
fitted with a simple power-law function with a probability of $0.23\left(\chi^{2}=16.3 / 13\right)$ and photon index $\Gamma=2.0 \pm 0.1$ and normalization factor at $250 \mathrm{GeV} f_{0}=(5.4 \pm 0.1) \times 10^{-11} \mathrm{~cm}^{-2} \mathrm{~s}^{-1} \mathrm{TeV}^{-1}$.

\section{EBL measurement}

We follow the procedure described in [19] for the likelihood ratio test. The absorption of the EBL is described as $e^{-\alpha \tau(E, z)}$ where $\tau(E, z)$ is the optical depth predicted by the model, which depends on the energy $E$ of the $\gamma$ rays and the redshift $z$ of the source. In this work we used the EBL model by Domínguez et al. [23]. The observed spectrum can be formed as:

$$
\left(\frac{d F}{d E}\right)_{o b s}=\left(\frac{d F}{d E}\right)_{i n t} \times \exp (-\alpha \times \tau(E, z))
$$

where $(d F / d E)_{\text {int }}$ is the intrinsic spectrum of the source. The models that describe the emission of HBLs, like 1ES 1011+496, predict a smooth and concave spectral energy distribution at VHE (e.g. [24]). With that, for the modeling of the intrinsic source spectrum we have used the same functions as in [14] and [19]: power-law (PWL), log-parabola (LP), power-law with exponential cut-off (EPWL), log-parabola with exponential cut-off (ELP) and power-law with superexponential cut-off (SEPWL). We have added the additional constraint that the shapes cannot be convex, i.e. the hardness of the spectrum cannot increase with energy.

To search for the imprint of the EBL on the observed spectrum, a scan over $\alpha$ was computed, varying the value from 0 to 2.5 . In each step of the scan, the model for the intrinsic spectrum was modified by the EBL absorption (scaled by $\alpha$ ) and then was passed through the response of the MAGIC telescopes. The result is compared to the actual observations (events recorded on- and offsource) using a Poissonian likelihood, which is computed for each value of alpha. This likelihood shows a maximum at a value $\alpha=\alpha_{0}$, indicating the EBL opacity scaling which achieves a best fit to the data. A likelihood ratio test was then performed to compare the no-EBL hypothesis $(\alpha=0)$ with the best-fit EBL hypothesis $\left(\alpha=\alpha_{0}\right)$. The test statistics $T S=2 \log \left(\mathscr{L}\left(\alpha=\alpha_{0} / \mathscr{L}(\alpha=0)\right)\right.$, according to Wilks' theorem, asymptotically follows a $\chi^{2}$ distribution with one degree of freedom.

The procedure described above is applied to the average spectrum during the flare, so that the event statistics is large and the Poissonian likelihood behaves close to its asymptotic limit. Despite the variable flux level during the flare, the EBL determination method should work properly as long as the average intrinsic spectrum in the observation period can be described with one of the tested parameterizations. A simple way to check the stability of the spectral shape is to de-absorb the night-wise spectra (in this case with the EBL model by [23]) and fit them to simple power-law functions. A fit to a constant photon index during the whole flare yields a $\chi^{2}$ of $23.5 / 16,(\mathrm{P}=0.1)$, hence, the data are compatible with stable spectral shape throughout the analyzed period.

Fig. 2 shows the $-2 \log \mathscr{L}$ probabilities for the five tested models. The model that gives the highest probability in the scanned range of $\alpha$ is the PWL. However, the selection of a PWL as model for the intrinsic spectrum would imply that all curvature in the observed spectrum come solely from the EBL absorption. Such strong assumption would result in an EBL "detection" at the $\sim 13 \sigma$ level. A more conservative approach is to choose the second-best function, the LP, which is actually the one providing the best fit for the no-EBL hypothesis. 

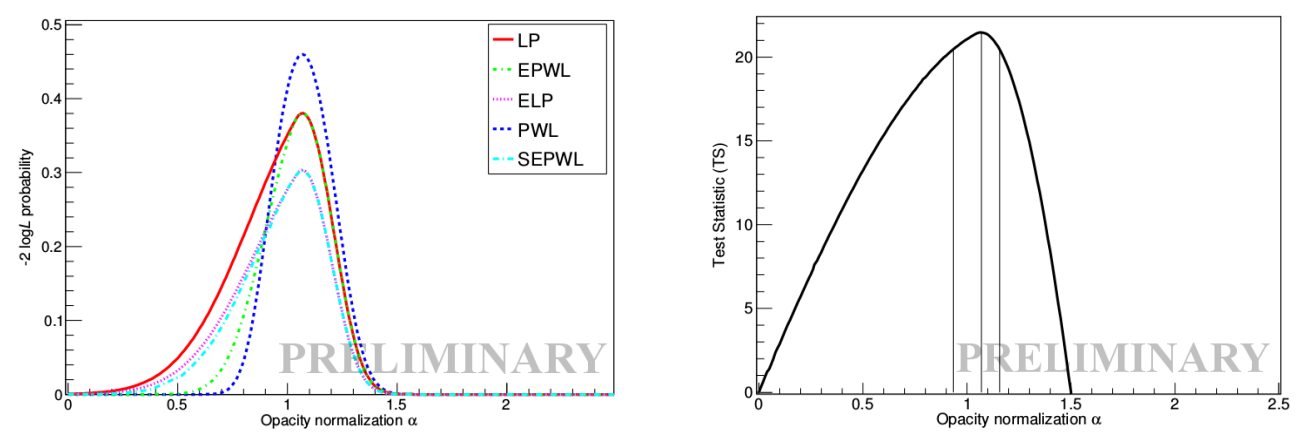

Figure 2: On the left, fit probability distributions for the average spectrum of the Feb-March flare of 1ES 1011+496 for the 5 models tested. PWL in blue (dashed line), LP in red (solid line), EPWL in green (dashdot line), ELP in pink (dotted line) and SEPWL in light blue (long dash-dot line). On the right, value of the test statistics distribution $\Delta \chi^{2}$ vs. the optical depth normalisation factor $\alpha$, under the assumption of a logparabolic intrinsic spectral shape. The vertical lines mark the maximum and the uncertainty corresponding to $1 \sigma$.

For the data sample from the 2014 February-March flare of 1ES 1011+496, and using the LP as our model for the intrinsic spectrum, the test statistics has a maximum of TS $=21.5$ at $\alpha_{0}=1.07_{-0.13}^{+0.09}$ (Fig. 2 right). This means that the EBL optical depth from the model of [23] scaled by the normalization factor $\alpha_{0}$ is preferred over the null EBL hypothesis with a significance of $4.6 \sigma$. Using the EBL model of Franceschini et al. [25] as template (as in [19]) the test statistic using the LP as model for the intrinsic spectrum has a maximum of TS=20.6 at $\alpha_{0}=1.14_{-0.14}^{+0.09}$, which is compatible with the result using Domínguez et al. model [23] within statistical uncertainty.

The MAGIC telescopes has a systematic uncertainty in the absolute light scale of $15 \%$ [21], coming mainly from the uncertainty is the imprecise knowledge of the atmospheric transmission. In order to assess how this uncertainty affects the EBL measurement, the calibration constants used to convert the pixel-wise digitized signals into photoelectrons were multiplied by a scaling factor (the same for both telescopes) spanning the range $-15 \%$ to $+15 \%$ in steps of $5 \%$. For each of the scaling factors the data were processed in an identical manner through the full analysis chain, starting from the image cleaning, and using in all cases the standard MAGIC MC for this observation period. In this way we try to asses the effect of a potential miscalibration between the data and the MC simulation.

For all scaled data samples, $-2 \log \mathscr{L}$ profiles for $\alpha$ between 0 and 2.5 were computed. We looked for the widest range in the statistical uncertainty around the minima $-2 \log \mathscr{L}$ values. This range in the uncertainty appeared as consequence of changing the light scale, therefore we can interpret it as a systematic uncertainty. Including this uncertainty, the measurement for the opacity normalization factor is $\alpha_{0}=1.07(-0.20,+0.24)_{\text {stat }+ \text { sys }}$.

\section{Discussion}

The relation of the $\gamma$-ray of energy $E_{\gamma}$ from the source (measured in the observed frame) and the EBL wavelength at the peak of the cross section for the photon-photon interaction is given by: 


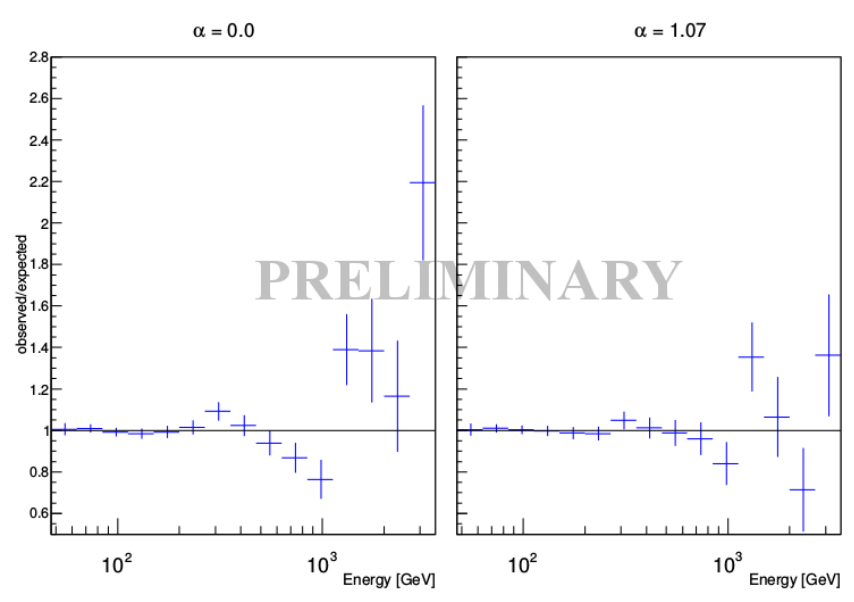

Figure 3: Ratio between the observed events and the expected events (signal+background) from the model of the intrinsic spectrum for two normalization values of the EBL optical depth, $\alpha=0$ to the left and $\alpha=1.07$ to the right, which corresponds to the normalization where the maximum TS was found. In both plots the line corresponding to a ratio $=1$ is shown.

$$
\lambda_{E B L}(\mu m)=1.187 \times E_{\gamma}(T e V) \times(1+z)^{2}
$$

where $z$ is equal or less than the redshift of the source. In Fig. 3 we show a comparison between the residuals computed for the null EBL hypothesis $\alpha=0$ (on the left) and the best fit EBL scaling $\alpha=1.07$ (on the right). The differences start to show at energies above $200 \mathrm{GeV}$, a region where the EBL introdues the feature that cannot be fitted by the log-parabola. We therefore calculate the approximate EBL wavelength range for which our conclusion is valid from the VHE range between 0.2 and 3.5 teV.

Based on the VHE range where the EBL imprint is visible in our data $(0.2-3.5 \mathrm{TeV})$, and taking into account the source redshift, we conclude that our measurement of the EBL density is valid in the range 0.24 to $4.15 \mu \mathrm{m}$. In Fig. 4 we show the contours from the statistical + systematic uncertainty of the EBL flux density, derived scaling up the EBL template model by Domínguez et al. [23] at redshift $z=0$. The wavelength coverage is in the so-called cosmic optical background (COB) part of the EBL, where we found the peak flux density $\lambda F_{\lambda}=12.27_{-2.29}^{+2.75} \mathrm{nW} \mathrm{m}^{-2} \mathrm{sr}^{-1}$ at 1.4 $\mu \mathrm{m}$, systematics included. From this measurement we found no evidence of exotic contributions to the EBL or anomalies in the propagation of $\gamma$ rays.

\section{Acknowledgments}

We would like to thank the Instituto de Astrofísica de Canarias for the excellent working conditions at the Observatorio del Roque de los Muchachos in La Palma. The financial support of the German BMBF and MPG, the Italian INFN and INAF, the Swiss National Fund SNF, the ERDF under the Spanish MINECO, and the Japanese JSPS and MEXT is gratefully acknowledged. This work was also supported by the Centro de Excelencia Severo Ochoa SEV-2012-0234, CPAN CSD2007-00042, and MultiDark CSD2009-00064 projects of the Spanish Consolider-Ingenio 2010 


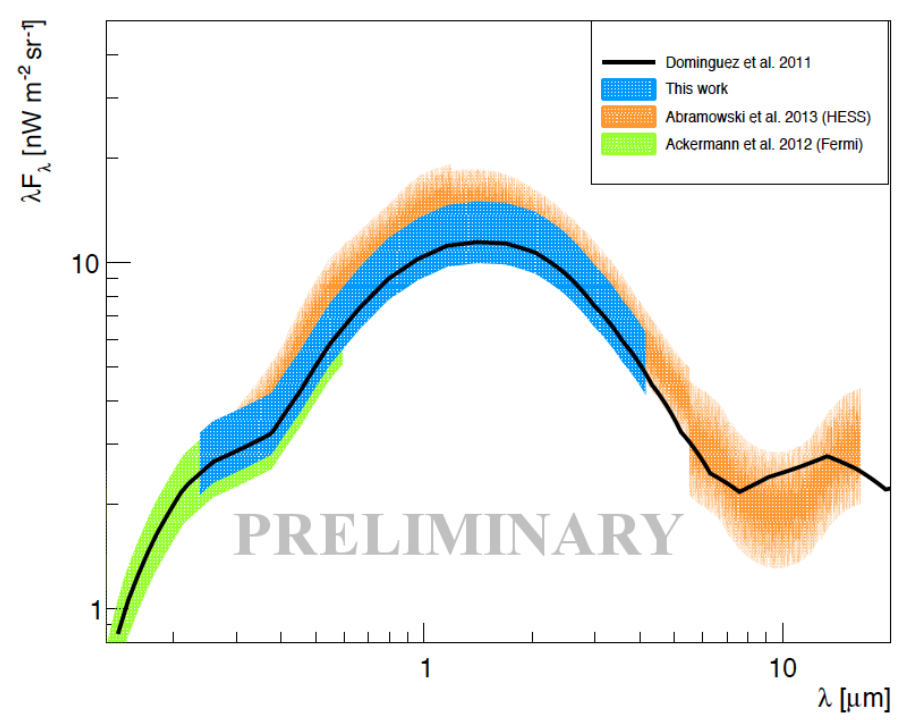

Figure 4: Extragalactic background light intensity versus wavelength at $z=0$. The solid black line is the EBL template model [23] that we used for our calculations. The blue shaded area spans the wavelength range for which our measurement is valid, scaled from the EBL template. The width of the shaded area includes the statistical and systematic uncertainties. The orange shaded area is the EBL measurement by [19] and the green shaded area is the measurement by [18].

programme, by grant 268740 of the Academy of Finland, by the Croatian Science Foundation (HrZZ) Project 09/176 and the University of Rijeka Project 13.12.1.3.02, by the DFG Collaborative Research Centers SFB823/C4 and SFB876/C3, and by the Polish MNiSzW grant 745/NHESS-MAGIC/2010/0.

\section{References}

[1] Albert, J. and Aliu, E. et al., Discovery of Very High Energy $\gamma$-Rays from $1 E S 1011+496$ at $z=0.212$, ApJ, 2007, 667, L21.

[2] Ansoldi, S. and Antonelli, A. et al., Multi-Wavelength Observations of the Blazar 1ES1011+496 in Spring 2008, In preparation, 2015.

[3] Ansoldi, S. and Antonelli, A. et al., Unprecedent insights in the blazar 1ES 1011+496 via MAGIC observations in 2011 and 2012 in combination with simultaneous MWL observations and polarimetry data, In preparation, 2015.

[4] Hauser, M. G. and Dwek, E., The Cosmic Infrared Background: Measurements and Implications, $A R A \& A, 2001,39,249$.

[5] Gould, R. J. and Schréder, G. P., Opacity of the Universe to High-Energy Photons, Physical Review, 1967, 155, 1408 .

[6] Madau, P. and Pozzetti, L., Deep galaxy counts, extragalactic background light and the stellar baryon budget, MNRAS 2000312 L9.

[7] Fazio, G. G. and Ashby, M. L. N. et al., Number Counts at $3 \mu \mathrm{m} \lambda 10 \mu \mathrm{m}$ from the Spitzer Space Telescope, ApJS 200415439. 
[8] Dole, H. and Lagache, G. et al., The cosmic infrared background resolved by Spitzer. Contributions of mid-infrared galaxies to the far-infrared background, A\&A 2006451417.

[9] Cooray, A. and Bock, J. J. and et al., First Star Signature in Infrared Background Anisotropies, ApJ, 2004, 606, 611.

[10] Fernandez, E. R. and Komatsu, E. and Iliev, I. T. and Shapiro, P. R., The Cosmic Near-Infrared Background. II. Fluctuations, ApJ, 2010, 710, 1089.

[11] Zemcov, M. and Smidt, J. et al., On the origin of near-infrared extragalactic background light anisotropy, Science, 2014, 346, 732.

[12] Stecker, F. W. and de Jager, O. C., Absorption of high energy gamma-rays by low energy intergalactic photons, Space Science Reviews, 1996, 75, 401.

[13] Aharonian, F. and Akhperjanian, A. G. et al., A low level of extragalactic background light as revealed by $\gamma$-rays from blazars, Nature, 2006, 440, 1018.

[14] Mazin, D. and Raue, M., New limits on the density of the extragalactic background light in the optical to the far infrared from the spectra of all known TeV blazars, A\&A, 2007, 471, 439.

[15] Georganopoulos, M. and Finke, J. D. and Reyes, L. C., A Method for Setting Upper Limits to the Extragalactic Background Light with Fermi-lat and TeV Observations of Blazars, ApJ, 2010, 714, L157.

[16] Orr, M. R. and Krennrich, F. and Dwek, E., Strong New Constraints on the Extragalactic Background Light in the Near- to Mid-infrared, ApJ, 2011, 733, 77.

[17] Meyer, M. and Raue, M. and Mazin, D. and Horns, D., Limits on the extragalactic background light in the Fermi era, A\&A, 2012, 542, A59.

[18] Ackermann, M. and Ajello, M. et al., The Imprint of the Extragalactic Background Light in the Gamma-Ray Spectra of Blazars, Science, 2012, 338, 1190.

[19] Abramowski, A. and Acero, F. et al., Measurement of the extragalactic background light imprint on the spectra of the brightest blazars observed with H.E.S.S., A\&A, 2013, 550, A4.

[20] Mazin, D. and Tescaro, D. et al., Upgrade of the MAGIC telescopes, ArXiv e-prints, 1410.5073, 2013.

[21] Aleksic, J. and Ansoldi, S. et al., The major upgrade of the MAGIC telescopes, Part II: The achieved physics performance using the Crab Nebula observations, ArXiv e-prints, 1409.5594, 2014.

[22] Zanin, R. and Carmona, E. et al., MARS, the MAGIC analysis and reconstruction software, Proc of the 33rd ICRC, Rio de Janeiro, 2013, 2, 773.

[23] Domínguez, A. and Primack, J. R. et al., Extragalactic background light inferred from AEGIS galaxy-SED-type fractions, MNRAS, 2011, 410, 2556.

[24] Tavecchio, F. and Maraschi, L. and Ghisellini, G., Constraints on the Physical Parameters of TeV Blazars, ApJ, 1998, 509,608.

[25] Franceschini, A. and Rodighiero, G. and Vaccari, M., Extragalactic optical-infrared background radiation, its time evolution and the cosmic photon-photon opacity, A\&A, 2008, 837. 